\title{
Vielfältige Hemmnisse überwinden
}

\author{
Sowohl das überbetriebliche Stoffstrommanagement als auch die Regionalisierung von Wirt- \\ schaftsaktivitäten sind in der theoretischen Diskussion über eine nachhaltige Entwicklung von \\ großer Bedeutung. In der betrieblichen Praxis werden Konzepte, die beide Ansäłze miteinander \\ verbinden, jedoch nur sehr vereinzelt umgesetzt. Der vorliegende Beitrag zeigt deshalb Umset- \\ zungskonzepte, Hemmnisse und daraus resultierenden Forschungsbedarf auf.
}

$\mathrm{D}$ ie Grundidee des Stoffstrommanagements ist die Transparenz sämtlicher, entlang einer Produktlinie anfallenden Stoff- und Materialströme. Durch diese ganzheitliche Betrachtungsweise soll die Grundlage zur Minderung des mit der Herstellung, Nutzung und Entsorgung von Produkten einhergehenden Verbrauches natürlicher Ressourcen und die Basis für eine nachhaltige Entwicklung geschaffen werden (1). Für die Erfassung und Minderung von Stoffströmen über die gesamte Produktlinie ist in der Regel eine unternehmensübergreifende Zusammenarbeit erforderlich. Eine solche Kooperation ist - unter anderem aufgrund der Möglichkeit zur Schließung von regionalen Stoffkreisläufen - im Sinne der Nachhaltigkeit insbesondere dann vorteilhaft, wenn die Kooperationspartner eine geringe räumliche Distanz zueinander aufweisen.

Obwohl regionale, überbetriebliche Stoffstrommanagementkonzepte in der theoretischen Diskussion eine große Relevanz für eine nachhaltige Unternehmensentwicklung besitzen, ist bei näherer Analyse festzustellen, dass sie in der Praxis bisher nur sehr vereinzelt realisiert werden (2). Im folgenden werden zunächst drei Typen regionaler Umsetzungskonzepte des überbetrieblichen Stoffstrommanagements - nämlich regionale Verwertungsnetzwerke, Industrieparks und Entsorgerparks - präsentiert und einander gegenübergestellt (siehe Tabelle 1).

\section{Regionale}

\section{Verwertungsnetzwerke}

Regionale Verwertungsnetzwerke, auch als Industriesymbiose oder Resource Recovery Networks bekannt, stellen eine überbetriebliche Zusammenarbeit zur Verknüpfung von Reststoffströmen zwischen mehreren Produktionsunternehmen dar. Ziel von Verwertungsnetzwer- men. Die in den USA vorherrschende Form der Eco-Industrial Parks zeichnen sich etwa dadurch aus, dass die beteiligten Unternehmen auf einem gemeinsamen Parkareal - in der Regel auf einem sanierten alten Industriegelände - angesiedelt werden. Dies wird von den zuständigen lokalen Behörden unterstützt und mögliche 0ptionen zur überbetrieblichen Kopplung von Stoff- und Energieflüssen finden Berücksichtigung. Die räumliche Entfernung zwischen den Unternehmen beträgt somit in der Regel höchstens wenige hundert Meter. Dies ermöglicht eine Kopplung leitungsgebundener Stoffströme, was bei größeren Entfernungen aufgrund des hohen Investitionsbedarfes häufig unrentabel ist. Hingegen befinden sich die Teilnehmer $\mathrm{eu}$ ropäischer Verwertungsnetzwerke zumeist nicht in unmittelbarer räumlicher Nähe. Dies gilt vor allem für Netzwerke, bei denen die Bündelung von Stoffströmen zur Nutzung von Größendegressionseffekten bei der Entsorgung im Vordergrund steht. Dort kann die Entfernung zwischen den Netzwerkteilnehmern bis zu 100 Kilometer betragen, sie werden daher auch als virtual Eco-Industrial Parks bezeichnet.

untergeordneter Bedeutung. Nur bei 2 von den 14 erfassten Verwertungsnetzwerken sind auch ökologische Zielsetzungen für die Entstehung der Netzwerke von Bedeutung.

Als Beispiele für regionale Verwertungsnetzwerke seien hier die Industriesymbiose in Kalundborg, das Verwertungsnetzwerk Steiermark, die Stoffstrommanagementnetzwerke Pfaffengrund und Rhein/Neckar (3) sowie die Demonstrationsnetzwerke in Baltimore, Cape Charles, Brownsville und Chattanooga in den USA genannt (4)

Verwertungsnetzwerke können bezüglich der räumlichen Distanz zwischen den Netzwerkakteuren sehr unterschiedliche Formen anneh-

\section{Industrieparks}

Ein produktionsorientiertes Umsetzungskonzept des überbetrieblichen Stoffstrommanagements, das eine unmittelbare räumliche Nähe zwischen den beteiligten Akteuren beinhaltet, ist das Konzept der Industrieparks. Diese firmieren auch unter den Bezeichnungen Zulieferparks, Industrial Supplier Parks oder Business Malls und sind durch eine Integration von Zulieferfirmen auf dem Werksgelände von Industrieunternehmen gekennzeichnet. Industrieparks eignen sich speziell für Produktionsstandorte mit einer durch

\begin{tabular}{l|l|l|l}
\multicolumn{4}{c}{ Tab. I:Klassifizierung regionaler Kooperationsformen des überbetrieblichen Stoffstrommanagements } \\
\hline Kriterium & \multicolumn{1}{|c|}{ Industrieparks } & Verwertungsnetzwerke & \multicolumn{1}{c}{ Entsorgerparks } \\
\hline Primäre Ziele & Verringerung der Fertigungstiefe, & Verwertung und Vermeidung & Reduktion von Logistikaufwand, \\
\hline der Kooperation & Reduktion von Logistikaufwand & von Rückständen & Erhöhung der Reststofftransparenz \\
\hline Netzwerkstruktur & Pyramidial & Polyzentrisch & Pyramidial \\
\hline Grundlegende & Produktionsunternehmen, & Produktionsunternehmen & Produktionsunternehmen, \\
\hline Netzwerkakteure & Zulieferunternehmen & & Entsorgungsunternehmen \\
\hline Räumliche & Werksgelände $<3 \mathrm{~km}$ & Je nach Form bis Zu $100 \mathrm{~km}$ & Werksgelände $<3 \mathrm{~km}$ \\
\hline Ausdehnung & & & \\
\hline Gradderhtegration & Sehr hoch & Gering bis mittel & Hoch \\
\hline Kommunikation, & Direkt, sehr intensiv & Direkt oder über Dritte, & Direkt, intensiv \\
\hline Information & & weniger intensiv & \\
\hline Funktions- & Produktionsorientiert & Produktionsorientiert, & Reduktionsorientiert \\
\hline orientierung & & Reduktionsorientiert & \\
\hline
\end{tabular}




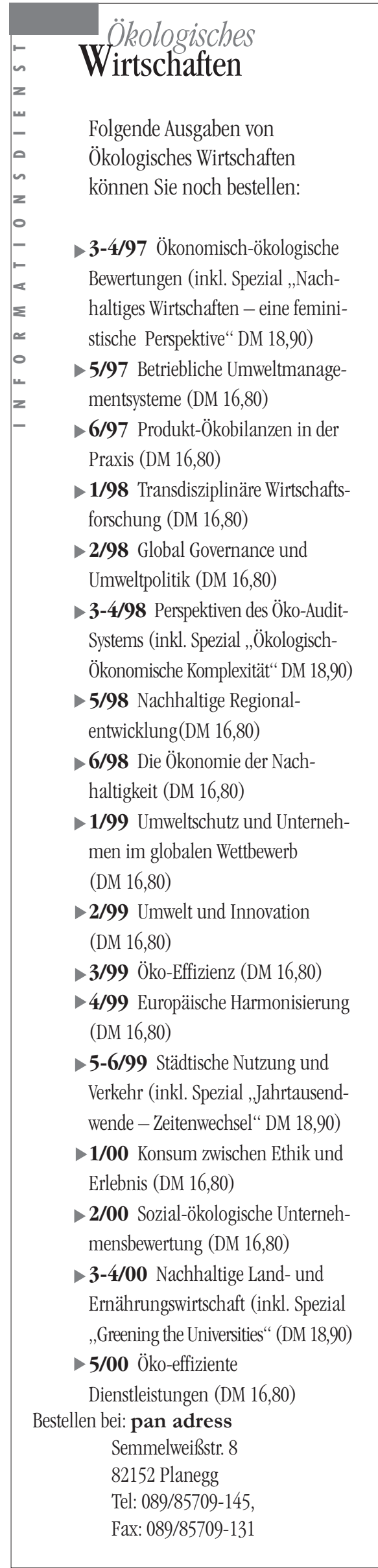

eine große Modellvielfalt bedingten hohen Produktkomplexität - beispielsweise für Montagewerke der Automobilindustrie. Der wesentliche Vorteil von Industrieparks ist in der räumlichen Nähe zwischen Produktions- und Zulieferunternehmen zu sehen. Sie ermöglicht eine passgenaue ,Just-in-Sequence“-Anlieferung voluminöser Bauteile und -gruppen über Förderbänder direkt an das Montageband. Beispiele finden sich in Rastatt (DaimlerChrysler), Neckarsulm (Audi), Valencia (Spanien, Ford-Werke) oder in Halewood (Großbritannien, Jaguar).

Obwohl mit der Realisierung von Industrieparks in der Regel primär eine Erhöhung der Kosteneffizienz des Produktionssystems erzielt werden soll, wie beispielsweise geringere Herstellungs-, Lager- und Nachbearbeitungskosten, kann dieses Konzept ebenso positive Auswirkungen auf die ökologische Nachhaltigkeitsdimension besitzen. So konnte im Fall des DaimlerChrysler-Werkes in Rastatt durch die Integration von zehn Zulieferfirmen auf dem Werksgelände eine Reduktion des beschaffungsbedingten Transportaufwands um ca. 12 Millionen LKW-Kilometer pro Jahr realisiert werden. Dabei wird vor allem das regionale Straßenverkehrsaufkommen deutlich gemindert.

\section{Entsorgerparks}

Entsorgerparks sind das reduktionsorientierte Pendant zu den produktionsorientierten Industrieparks. Das Konzept beinhaltet eine Auslagerung entsorgungslogistischer Aktivitäten auf externe Entsorgungsdienstleistungsunternehmen, die in einem Entsorgerpark auf dem Werksgelände angesiedelt sind. Durch die unmittelbare räumliche Nähe des Entsorgerparks zur Produktionsstätte besitzen diese - neben der Möglichkeit zur Reduktion von Entsorgungskosten - ein beachtliches Potenzial zur Verbesserung der ökologischen Dimension einer nachhaltigen Unternehmensentwicklung, etwa durch

- ein Schließen von Stoffkreisläufen auf dem Werksgelände,

- eine Erhöhung der Transparenz anfallender Stoffströme,

- eine Minderung von Reststoffhandling und

eine Reduktion der mit der Entsorgung verbundenen Transportleistungen.

\section{Hemmnisse}

Die Leitideen des überbetrieblichen Stoffstrommanagements und des regionalen Wirtschaftens sind in der betrieblichen Praxis, insbesondere im Rahmen von reduktionsorientierten Kooperationen des überbetrieblichen Stoffstrommanagements, noch nicht auf breiter Front umgesetzt worden. Diese Tatsache kann mit der Existenz vielfältiger betriebsinterner und betriebsübergreifender Hemmnisse erklärt werden.

$\mathrm{Zu}$ den betriebsinternen Hemmnissen gehört zum Beispiel die oft untergeordnete Bedeutung des Nachhaltigkeitsziels in der persönlichen sowie unternehmensweiten Zielhierarchie. Darüber hinaus besteht häufig ein Mangel an Promotoren sowie eine mangelnde Bekanntheit der regionalen Unsetzungskonzepte innerhalb von Unternehmen. Diese Tatsache hat sich auch im Rahmen einer Befragung von 25 Industrievertretern bestätigt (2).

Ein zentrales betriebsübergreifendes Hemmnis des überbetrieblichen Stoffstrommanagements ist die Notwendigkeit zur Offenlegung von Information. So ist bei der Untersuchung der Vorteilhaftigkeit möglicher Konzepte eine Analyse des Ist-

\section{punkt-um.net}

\section{Ihre Internet-Plattform zum Infodienst punkt.um für Informationen zu Nachhaltigkeit und Ökologie}

Aktuelle Termine - News aus dem Internet - Website-Datenbank

Ökomedia: Abstracts-Datenbank zu 70.000 Zeitschriftenartikeln und Bücher seit 1987

Sie finden uns unter: http://www.punkt-um.net 
Zustandes der betriebsinternen Situation erforderlich. In diesem Zusammenhang erweist sich insbesondere die Offenlegung von ökonomischen Daten als kritisch.

Ein weiteres betriebsübergreifendes Hemmnis stellt ein mögliches gegenseitiges Abhängigkeitsverhältnis zwischen den Kooperationspartnern dar. Dies gilt insbesondere für den Fall, dass die Realisierung des überbetrieblichen Konzeptes mit einem hohen Investitionsbedarf verbunden ist und die Wirtschaftlichkeit der Investition wesentlich von der Leistungserbringung des Partnerunternehmens abhängt. Beispielsweise hängt bei der Investition in Anlagen zur Kopplung von Stoffströmen innerhalb von Verwertungsnetzwerken die Gewinnträchtigkeit wesentlich von der Qualität, Quantität und Kontinuität der zu koppelnden Stoffströme ab. Deren Einhaltung liegt jedoch in der Regel außerhalb des Einflussbereiches des abnehmenden Unternehmens.

Schließlich hemmt die Existenz von Transaktionskosten - beispielsweise zur Identifikation möglicher Partner, zur Vertragsgestaltung bzw. zur Koordination der Stoffströme - die Umsetzung derartiger Kooperationsformen.

\section{Was ist zu tun?}

Zur Überwindung der skizzierten Barrieren ist die Kommunikation des Nachhaltigkeitsbegriffes sowie des möglichen Beitrages regionaler überbetrieblicher Stoffstrommanagementkonzepte für eine nachhaltige Unternehmensentwicklung unabdingbar. Darüber hinausgehend sind weitere Forschungsarbeiten bezüglich der Organisationsformen, Instrumente und Methoden zur Unterstïtzung erforderlich. Dies gilt aufgrund bestehender Defizite vor allem für die reduktionsorientierten Konzepte der Verwertungsnetzwerke und Entsorgerparks.

Für Verwertungsnetzwerke gilt es Konzepte zur organisatorischen Ausgestaltung zu entwickeln, insbesondere im Hinblick auf

die Sicherung der Qualität und Kontinuität der gekoppelten Stoffflüsse,

die Sicherung der Netzwerkkontinuität,

den Aufbau eines Vertrauensverhältnisses zwischen den beteiligten Akteuren,

- die organisatorische Einbindung des Netzwerkes in die Netzwerkumgebung,

- die Flexibilität des Netzwerkes bei der Reaktion auf unvorhergesehene Ereignisse und der Verlängerung der von der Industrie geforderten möglichst kurzen Amortisationszeiten durch die Einbindung weiterer Akteure zur Finanzierung (Contracting).

Für eine Realisierung von Entsorgerparks sind insbesondere die im Rahmen der bereits bestehenden Industrieparks generierten organisatorischen Umsetzungskonzepte auf die Entsorgungsseite zu übertragen und erstmalig anzuwenden.

\section{Ausblick}

Zusammenfassend ist festzustellen, dass die vorgestellten Konzepte des regionalen, überbetrieblichen Stoffstrommanagements aufgrund vielfältiger Hemmnisse nur sehr vereinzelt umgesetzt werden. Insbesondere für eine Realisierung der reduktionsorientierten Kooperationsformen ist zumeist ein externer Anstoß erforderlich. Mit dem Ziel einer weiteren Verbreitung der vorgestellten Konzepte ist deshalb auch zukünftig eine Unterstïtzung durch forschungsfördernde Institutionen erforderlich, wie sie derzeit beispielsweise im Rahmen der Initiative Modellprojekte für nachbaltiges Wirtschaften des Forschungsministeriums erfolgt (5).

\section{Anmerkungen}

(1) Vgl. auch Brickwedde, F. (Hrsg.): Stoffstrommanagement Herausforderung für eine nachhaltige Entwicklung. Tagungsband zur 4. internationalen Sommerakademie, St. Marienthal, Deutsche Bundesstiftung Umwelt. Osnabrück 1999.

(2) Die Erkenntnisse stammen aus einem vom Bundesministerium für Bildung und Forschung (BMBF) geförderten Projekt. Vgl. Rentz, 0. et al.: Vordringliche Aktion des Bundesministeriums für Bildung und Forschung (BMBF): Produkte und Prozesse mit dem Ziel der Nachhaltigkeit: Endbericht zum Teilprojekt „Regionalisierung und lokale Kreislaufwirtschaft", IIP, Karlsruhe 2000.

(3) Wietschel, M./ Rentz, 0.: Verwertungsnetzwerke im Vergleich zu anderen Unternehmensnetzwerken. In: Liesegang, D.G./ Sterr, T./ Ott, T. (Hrsg.): Regionale Stoffstrommanagementnetzwerke. Tagungsband zum Fachkongress, IUWA, Heidelberg 2000.

(4) Vgl. http://www.cfe.cornell.edu/WEl/EIDP/design.html. (5) Vgl. http://www.nachhaltig.org.

Die Autoren
Sven Graehl ist wissenschaftlicher Mitarbeiter,
Dr. Wolf Fichtner ist wissenschaftlicher Assistent und
Prof. Dr. Otto Rentz ist Direktor des Instituts für Indus-
triebetriebslehre und Industrielle Produktion (IIP)
an der Universität Karlsruhe (TH).
Kontakt:IIP, Hertzstr. 16, 76187 Karlsruhe.
Tel. 0721/ 608-4690,
E-mail: sven.graeh|@wiwi.uni-karlsruhe.de

\section{politische ökologie a-z}

\section{Wählen Sie aus unserem Gesamtverzeichnis}

pö 52 Geduldsspiel Nachhaltigkeit -Agenda 21 als Leitfaden für das nächste Jahrhundert

pö 53 Wechsel ohne Deckung - Über den Zusammenhang von Geld und Umwelt

pö 54 Nachhaltiges Arbeiten - Ein Weg aus der Beschäftigungs- und Umweltkrise?

pö 55 Gratwanderung - zwischen Autarkie und Globalisierung: Die Alpen als Vorreiter für ein regionales Wirtschaften

pö 56 Endspurt - Die Ökologische Steuerreform vor dem Durchbruch?

pö 57/58 Von der Zeitnot zum Zeitwohlstand Auf der Suche nach den rechten Zeitmaßen

pö 59 Wa(h)re Wildnis - Reise Special ' 99

pö 60 Risiko

pö 61 Wechselstrom - Anleitung für eine neue Energiepolitik

pö 62 Stoffwechsel - Naturstrategien als Vorbild für Nach-haltigkeit

pö 63/64 Nachhaltigkeit öffne dich! Kommunikation eines Leitbildes inklusive Literatur Spezial 2000 pö 65 e_cotopia - von ökologischen visionen zu virtuellen realitäten

pö 66 jenseits des wachstums

(Einzelhefte 19,80 DM, Doppelausgaben 24,80)

\section{Sonderhefte}

pö Sonderheft 8 Zeit-Fraß - Zur Ökologie der Zeit in Land-wirtschaft und Ernährung

pö Sonderheft 9 Handwerk als Schlüssel für eine zukunftsfähige Wirtschaft

pö Sonderheft 10 Bodenlos - Zum nachhaltigen Umgang mit Böden

pö Sonderheft 11 Wege aus der Wachstumsfalle - Perspektiven einer zukunftsfähigen Wirtschaftsund Lebensweise

pö Sonderheft 12 Schnittmenge Mensch - Bildung für nachhaltige Entwicklung als neue Lernkultur

(Sonderhefte zum Preis von je 19,80 DM)

PÖ-Reise-Special 2000 - Exponierte Zukunft 12,80 DM

PÖ-Literatur Special - Umweltbücher 2000 $12,80 \mathrm{DM}$

Abo für sechs Ausgaben: DM 98,00, (Institutionen DM 148,-), ermäßigt: DM 78,00 (ohne Versandkosten)

Eine Übersicht aller noch erhältlichen Ausgaben finden Sie auch auf unserer Internetseite:

www.oekom.de 
(c) 20I0 Authors; licensee IÖW and oekom verlag. This is an article distributed under the terms of the Creative Commons Attribution Non-Commercial No Derivates License (http://creativecommons.org/licenses/by-nc-nd/3.o/), which permits unrestricted use, distribution, and reproduction in any medium, provided the original work is properly cited. 